\title{
Effect of dynamical friction on interchange motion of plasma filaments
}

\author{
R. Kube and O. E. Garcia ${ }^{\text {a) }}$ \\ Department of Physics and Technology, University of Troms $\phi, N 9037$ Troms $\phi$, Norway and MIT Plasma \\ Science and Fusion Center, Cambridge, Massachusetts 02139, USA
}

(Received 11 January 2012; accepted 23 March 2012; published online 26 April 2012)

\begin{abstract}
Theory and numerical simulations are presented for interchange motion of plasma filaments in the presence of dynamical friction and allowing large relative filament amplitudes. When friction is negligible, the filament velocity is proportional to the square root of gravity and its cross-field size. For strong friction, the filament velocity is independent of the cross-field size, proportional to gravity, and inversely proportional to the friction coefficient. In this frictional regime, the filament moves a large distance with nearly constant velocity and shape. The transition between these velocity scaling regimes and the amplitude dependence are revealed. The results presented here complement previous theories for irregularities in the equatorial ionosphere and are in excellent agreement with recent experiments on simply magnetized toroidal plasmas. The relevance to blob-like structures in the scrape-off layer of magnetically confined plasmas is also discussed. (C) 2012 American Institute of Physics. [http://dx.doi.org/10.1063/1.4704793]
\end{abstract}

\section{INTRODUCTION}

Plasma filament structures that are elongated along the magnetic field and localized in the drift plane perpendicular to the field have been found to strongly influence the transport of particles and heat in magnetized plasmas as diverse as the equatorial ionosphere, ${ }^{1-5}$ basic laboratory experiments, ${ }^{6-10}$ and magnetically confined fusion plasmas. ${ }^{1-15}$ In all these cases, buoyancy or magnetic field curvature causes charge polarization and interchange motions across the magnetic field lines. In this contribution we investigate the effect of dynamical friction on the dynamics of such filament structures.

In the equatorial F-layer ionosphere, field-aligned irregularities are frequently produced on the night side, resulting in strong plasma depletions that due to buoyancy propagate radially outwards and may cause radio wave scintillation that degrades communication and navigation systems. ${ }^{3}$ These structures, often referred to as equatorial spread-F (ESF) bubbles, can propagate beyond the peak of the F-layer ionosphere. Dynamical friction, resulting from collisions between plasma and neutral gas particles, strongly influences the motion of these plasma filaments. ${ }^{4,5}$ A frictional regime was identified, in which the irregularities move across the field lines with a velocity that is proportional to gravity, inversely proportional to the ion neutral collision frequency and independent of the cross-field filament size. The theory and numerical simulations presented here will be shown to support and extend previous theories for the evolution of ESF irregularities. However, we do not attempt make a detailed description of ESF irregularities, which is further complicated by for example B-parallel dynamics, multiple ion species and coupling to neutral dynamics. ${ }^{1-3}$

Recently, several laboratory experiments have been done on filament motion in toroidally magnetized plasmas. In the Versatile Toroidal Facility (VTF) device, an isolated plasma filament was produced at the high field side, and the

${ }^{a)}$ Electronic mail: odd.erik.garcia@uit.no. radial motion recorded by a large probe array. ${ }^{8}$ The filament developed a mushroom-like cap shape and a dipole electrostatic potential structure. The radial velocity was found to be inversely proportional to the neutral gas pressure and independent of the cross-field filament size for the parameter regimes investigated. We present results from numerical simulations that compare favourably with these experimental measurements, showing that the filament velocity depends on the ion neutral collision frequency $\nu_{\mathrm{i}}$ in the same way as for equatorial F-region irregularities discussed above.

In magnetically confined plasmas, blob-like structures frequently form in the edge region and propagate radially through the scrape-off layer. This causes enhanced levels of plasma wall interactions that may limit the performance and operation of fusion reactors. ${ }^{16-20}$ Plasma recycling on material surfaces can result in large neutral gas particle densities in the scrape-off layer and significant impact of ion neutral collisions on the motion of blob-like structures. Moreover, for structures ballooned on the low field side, the parallel dynamics may result in an effective dynamical friction with the friction coefficient $\nu_{\mathrm{i}}$ given by $C_{\mathrm{s}} / L$ in the electrostatic regime $^{21-23}$ and $C_{\mathrm{A}} / L$ in the electromagnetic regime, ${ }^{24,25}$ where $C_{\mathrm{s}}$ and $C_{\mathrm{A}}$ are the acoustic and Alfvén speeds, respectively, and $L$ the parallel structure length. The following investigations on the role of dynamical friction may therefore apply to these cases as well. The origin of effective dynamical friction is discussed in some detail in the appendix.

This paper is organized as follows. In Sec. II we present the reduced model equations, analytical solutions, and velocity scalings. In Sec. III we present results from numerical simulations of the filament dynamics, addressing the role of dynamical friction. A discussion of the simulation results is given in Sec. IV, revealing the size and amplitude dependence of the filament velocity. In Sec. V we discuss how these results compare to basic laboratory experiments on filament motion on the VTF device. Finally, in Sec. VI we present a brief summary and conclusions. 


\section{MODEL EQUATIONS}

The dynamical evolution of filament structures is governed by the dominant current channels, which are determined by the filament size, amplitude, and plasma parameters. This is described by the one-fluid momentum equation including buoyancy and dynamical friction

$$
\rho\left(\frac{\partial}{\partial t}+\mathbf{V} \cdot \nabla\right) \mathbf{V}=-\nabla P+\mathbf{J} \times \mathbf{B}+\rho \mathbf{g}-\rho \nu_{\mathrm{i}} \mathbf{V},
$$

where $\rho$ is the mass density, $P$ the plasma pressure, and $\mathbf{g}$ gravity. The friction coefficient $\nu_{\mathrm{i}}$ is in the following assumed to be constant. In the case of ion neutral collisions giving rise to dynamical friction, the analysis is performed in the reference frame of the neutrals.

The momentum equation can be used to obtain explicit expressions for the cross-field currents in the case of lowfrequency dynamics. The electric current density due to gravitational particle drifts is then given by

$$
\mathbf{J}_{g}=\frac{\rho}{B} \mathbf{g} \times \mathbf{b}
$$

In an inhomogeneous plasma this causes charge polarization in the direction perpendicular to gravity and the magnetic field, similar to the baroclinic generation of vorticity in ordinary fluids. ${ }^{26}$

For non-uniformly magnetized plasmas, effective gravity arises from magnetic field curvature and inhomogeneity. In this case, the diamagnetic current $\mathbf{J}_{d}=\mathbf{b} \times \nabla P / B$ is compressible and gives rise to charge polarization similar to that described by Eq. (2), where the effective gravity is given by $^{27,28}$

$$
\mathbf{g}=-C_{\mathrm{s}}^{2}\left(\mathbf{b} \cdot \nabla \mathbf{b}+\nabla_{\perp} \ln B\right),
$$

where $C_{\mathrm{s}}=(P / \rho)^{1 / 2}$ is the acoustic speed. This has been identified as the mechanism for radial motion of blob-like structures in the scrape-off layer of magnetically confined plasmas. $^{29-33}$

Assuming electrostatic perturbations, the lowest order cross-field motion is given by the electric drift $\mathbf{b} \times \nabla \phi / B$, where $\phi$ is the electrostatic potential. Further neglecting B-parallel motions due to flute ordering gives the electric current density caused by polarization drifts

$$
\mathbf{J}_{p}=-\frac{\rho}{B}\left(\frac{\partial}{\partial t}+\frac{1}{B} \mathbf{b} \times \nabla \phi \cdot \nabla\right) \frac{\nabla_{\perp} \phi}{B} .
$$

Plasma inertia enters through this current channel, and it is well known to be of crucial importance for non-linear plasma dynamics in general and turbulent flows in particular.

Finally, there is an electric current density due to dynamical friction, which in the electrostatic limit is given by

$$
\mathbf{J}_{\nu}=-\frac{\rho \nu_{\mathrm{i}}}{B^{2}} \nabla_{\perp} \phi
$$

In weakly ionized plasmas, ion collisions with neutral particles result in currents along the electric field that prevent charge build-up by buoyancy. In the lower part of the ionosphere, the ion neutral collision frequency can be so large that the ions are unmagnetized. In this case the gravitational current is balanced by the friction current rather than polarization currents.

Neglecting for the moment collisional diffusion and viscosity, a closed model that comprises all the current channels discussed above is given by

$$
\begin{gathered}
\left(\frac{\partial}{\partial t}+\frac{1}{B} \mathbf{b} \times \nabla \phi \cdot \nabla\right) \rho=0, \\
\nabla \cdot\left[\rho\left(\frac{\partial}{\partial t}+\frac{1}{B} \mathbf{b} \times \nabla \phi \cdot \nabla\right) \frac{\nabla_{\perp} \phi}{B}+\nu_{\mathrm{i}} \rho \nabla_{\perp} \phi\right] \\
+\mathbf{b} \times \mathbf{g} \cdot \nabla \rho=0 .
\end{gathered}
$$

It is clear that the gravitational current will lead to charge build-up within a plasma filament structure and that the balance of ion polarization and friction currents will determine the dynamical evolution of the filament.

In the ideal limit where friction can be neglected, there is an inertial velocity scaling for an isolated filament structure given by

$$
V \sim(g \ell)^{1 / 2}
$$

where $\ell$ is the cross-field filament size. This scaling, predicting a velocity increasing with the square of the size, follows from dimensional analysis and has previously been demonstrated by numerical simulations. ${ }^{31-33}$ The temporal scale for the filament evolution is the ideal interchange rate $\gamma=(g / \ell)^{1 / 2}$.

We introduce a rectangular coordinate system with gravity in the $x$-direction and the magnetic field in the $z$-direction. Assuming separation of variables, it is readily found that the model (6a) and (6b) has an analytical solution describing radial motion of any wave form

$$
\begin{gathered}
\rho(\mathbf{x}, t)=\Theta\left(x-g t / \nu_{\mathrm{i}}, y\right) \Psi(y), \\
\phi(\mathbf{x}, t)=-B g y / \nu_{\mathrm{i}},
\end{gathered}
$$

where $\Theta$ and $\Psi$ are arbitrary functions that describe the initial filament shape in the drift plane. This solution represents a structure moving along the direction of gravity with terminal velocity

$$
V \sim \frac{g}{\nu_{\mathrm{i}}}
$$

independent of the cross-field filament size. It should be noted that there is no vorticity and hence no plasma inertia associated with this analytical solution.

The frictional velocity scaling given by Eq. (9) is expected to prevail when the friction coefficient is large. The transition between the inertial and frictional regimes will occur when $\nu_{\mathrm{i}}$ is of the same order of magnitude as the ideal interchange rate $\gamma$. This defines a frictional length scale given by $g / \nu_{\mathrm{i}}^{2}$. For cross-field filament sizes $\ell \ll g / \nu_{\mathrm{i}}^{2}$, we expect the inertial velocity scaling given by Eq. (7) to hold. For filament sizes $\ell \gg g / \nu_{\mathrm{i}}^{2}$, the frictional velocity scaling given by Eq. (9) is expected. In the Sec. III we present results from 
numerical simulations that connect these two velocity scaling regimes and elucidate the amplitude dependence.

\section{NUMERICAL SIMULATIONS}

In order to analyze the radial interchange motion of isolated plasma filaments, we investigate a reduced two-field model describing the evolution of the particle density $n$ and electric drift vorticity $\Omega=\nabla_{\perp}^{2} \phi / B$. Spatial scales are normalized with a characteristic cross-field filament size $\ell$ and temporal scales with the corresponding ideal interchange rate $\gamma$. The non-dimensional model is then written in the form

$$
\begin{gathered}
\frac{d \ln n}{d t}=\kappa \nabla_{\perp}^{2} \ln n+\kappa\left(\nabla_{\perp} \ln n\right)^{2}, \\
\frac{d \Omega}{d t}+\frac{\partial \ln n}{\partial y}+\nu \Omega=\mu \nabla_{\perp}^{2} \Omega,
\end{gathered}
$$

where $d / d t=\partial / \partial t+\hat{\mathbf{z}} \times \nabla \phi \cdot \nabla$, the normalized friction coefficient is given by $\nu=\nu_{\mathrm{i}} / \gamma$, and we have added crossfield collisional diffusion and viscosity with normalized diffusion coefficients $\kappa$ and $\mu$, respectively. In the remaining part of this paper we use these non-dimensional units unless otherwise explicitly stated.

In the following we present results from numerical simulations of the model (10a) and (10b). In order to investigate the dynamics of an isolated plasma filament, we write the particle density as

$$
\frac{n}{N}=1+\frac{\Delta n}{N} \theta
$$

where $N$ is a homogeneous background particle density, $\Delta n / N$ is the relative filament amplitude, and the spatial variation is given by $\theta$. For the numerical simulations, $\theta$ is initially taken to be $\mathrm{be}^{31-33}$

$$
\theta(\mathbf{x}, t=0)=\exp \left(-\frac{1}{2} \mathbf{x}^{2}\right)
$$

There is initially no fluid flow, $\phi(\mathbf{x}, t=0)=0$, so the collective motions arise from charge polarization due to particle drifts across the magnetic field, which is described by the interchange term in the vorticity Eq. (10b). Note that due to the absence of free-energy sources, the asymptotic state is that of thermodynamic equilibrium with the initial plasma blob structure distributed homogeneously in space.

The model has been solved on a biperiodic square domain using a standard Fourier Galerkin method with size up to $100^{2}$ and the number of grid points up to $8192^{2}$. The normalized diffusion coefficients $\kappa$ and $\mu$ are both set to $10^{3}$, which ensures that we are in the ideal regime even for relative filament amplitudes as small as $10^{2}$ in the absence of friction. ${ }^{31,32}$

To quantify the radial motion of the plasma filament, we define the radial center of mass position and velocity

$$
X(t)=\frac{1}{2 \pi} \int d \mathbf{x} x \theta, \quad V(t)=\frac{d X}{d t},
$$

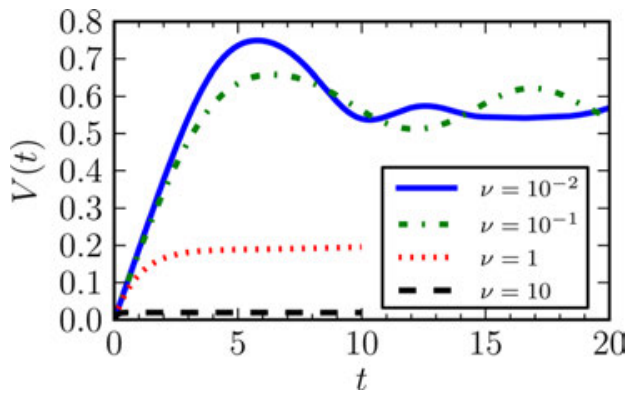

FIG. 1. Evolution of the radial centre of mass velocity for $\Delta n / N \quad 1$ and various friction coefficients.

where the integration is over the whole simulation domain and $\theta$ is defined in Eq. (11). From Eq. (10a) it can be shown that the radial centre of mass velocity is equal to the total radial particle flux caused by the collective motions.

The dynamical evolution of the radial centre of mass velocity for $\Delta n / N=1$ and various friction coefficients is presented in Fig. 1. Following an initial period of fast acceleration, the filament reaches a maximum velocity after approximately 6 ideal interchange times for $\nu<1$. It has previously been shown that the evolution at later times in the ideal limit is dominated by the development of small scale flows and dispersion of the filament structure that sensitively depend on the collisional diffusion coefficients. ${ }^{31-33}$ For friction coefficients of order unity and larger the centre of mass velocity remains approximately constant for a long time after the initial acceleration. The terminal radial velocity decreases with increasing friction coefficient.

The filament dynamics also depends on the relative amplitude. In Fig. 2 we show the dynamical evolution of the radial centre of mass velocity for $\nu=1$ and various filament amplitudes. For very large amplitudes there is a pronounced peak for the radial velocity, while the velocity is nearly constant for a long time in the case of small to intermediate filament amplitudes.

Contour plots of the particle density with super-imposed electrostatic potential iso-lines are presented for various values of the friction coefficient in Fig. 3 for $\Delta n / N=1$ and in Fig. 4 for $\Delta n / N=10^{3}$ at the time of maximum radial centre of mass velocity. For large friction coefficients, these structures pertain for a long time during the subsequent motion of the filaments. It is clearly seen that the filament structure develops an asymmetric shape with a steep front and a

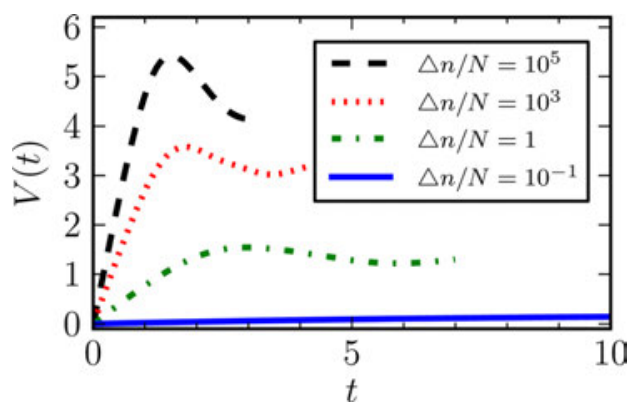

FIG. 2. Evolution of the radial centre of mass velocity for $\nu \quad 1$ and various filament amplitudes. 

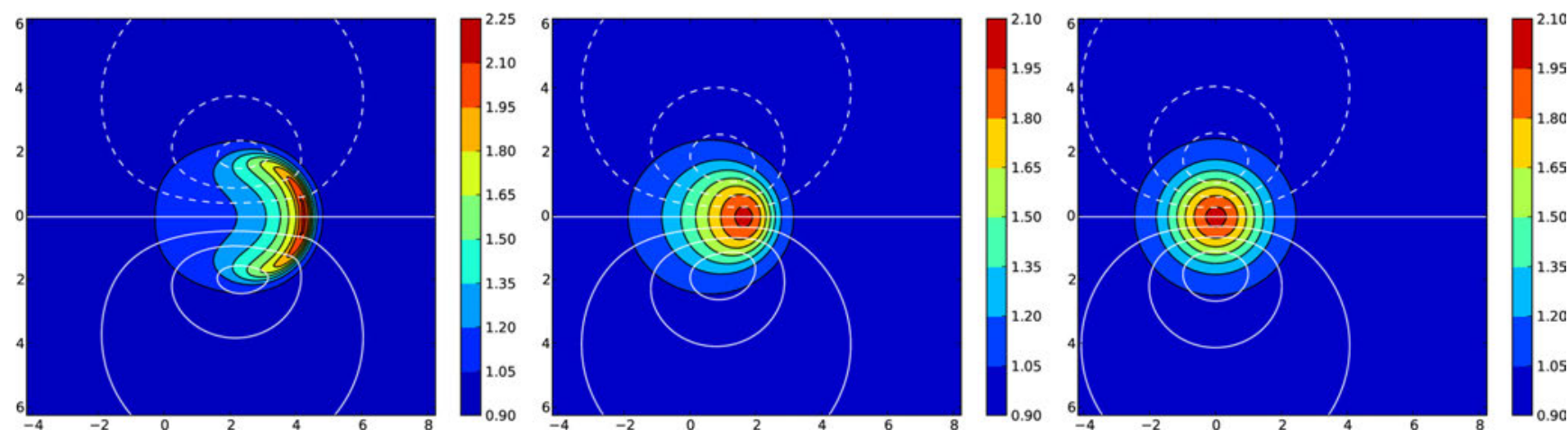

FIG. 3. Particle density contour plots with super imposed electrostatic potential iso lines for $\Delta n / N \quad 1$ and $\nu 10^{1}$ (left), 1 (middle), and 10 (right) at time $t 6$.

trailing wake for small friction coefficients. This radial asymmetry is reduced, resulting in a more circular shaped blob-structure, when either the amplitude or friction coefficient becomes larger. The filament velocity is very small when the friction coefficient is larger than unity, even for very large filament amplitudes.

In Fig. 5 we present the scaling of the maximum radial centre of mass velocity as function of the friction coefficient for various filament amplitudes. For small $\nu$ the velocity is independent of friction while for large $\nu$ the velocity is inversely proportional to the friction coefficient. Similarly, Fig. 6 shows the maximum velocity as function of relative amplitude for various friction coefficients. The filament velocity clearly increases with the relative amplitude. For small filament amplitudes and friction coefficients we recover the square root amplitude scaling $V \sim(\Delta n / N)^{1 / 2}$ (Refs. 3133 ). This scaling breaks for filament amplitudes of order unity or larger and transits into a regime where the filament velocity is only weakly dependent on the amplitude for $\Delta n / N \gg 1$. Further discussion of the velocity dependence on amplitude is given in the following section.

\section{DISCUSSION AND INTERPRETATION}

Applying an order of magnitude estimate with $\partial / \partial t$ $\sim \Omega \sim V$, the vorticity equation (10b) gives $V^{2}-d_{1} \Delta n$ $/(N+\Delta n)+d_{2} \nu V=0$, where $d_{1}$ and $d_{2}$ are fit coefficients that depend on the filament amplitude. The positive root for the filament velocity is given by

$$
V=-\frac{d_{2} \nu}{2}+\left(\frac{d_{2}^{2} \nu^{2}}{4}+d_{1} \frac{\Delta n}{N+\Delta n}\right)^{1 / 2} .
$$

Fitting this model to the numerical simulation data for a fixed filament amplitude, we find the fit parameters $d_{1}$ and $d_{2}$. The fit of Eq. (14) to the simulation data is presented by the solid lines in Fig. 5 and the fit coefficients $d_{1}$ and $d_{2}$ as function of the relative filament amplitude in Fig. 7. The simple order of magnitude estimate clearly yields a good description of the simulation data. For small friction coefficients, $\nu \ll 1$, Eq. (14) gives $V \approx\left[d_{1} \Delta n /(N+\Delta n)\right]^{1 / 2}$ independent of $\nu$ as observed in Fig. 5. For large friction coefficients, $\nu \gg 1$, we get $V \approx d_{1} \Delta n / d_{2} \nu(N+\Delta n)$, inversely proportional to the friction coefficient as seen in Fig. 5.

When Eq. (14) is written in dimensional units, the filament velocity dependence on the cross-field size becomes explicit

$$
\frac{\nu_{i} V}{g}=\frac{d_{2}}{2} \frac{\nu_{i}^{2} \ell}{g}\left[-1+\left(1+\frac{4 d_{1}}{d_{2}^{2}} \frac{g}{\nu_{i}^{2} \ell} \frac{\Delta n}{N+\Delta n}\right)^{1 / 2}\right] .
$$

There is a characteristic size given by $g / \nu_{\mathrm{i}}^{2}$ that separates the two velocity scaling regimes. For small filament sizes, $\ell \ll g / \nu_{\mathrm{i}}^{2}$, we find the inertial velocity scaling given by Eq. $(7)^{31-33}$

$$
V \approx\left(d_{1} g \ell \frac{\Delta n}{N+\Delta n}\right)^{1 / 2}
$$
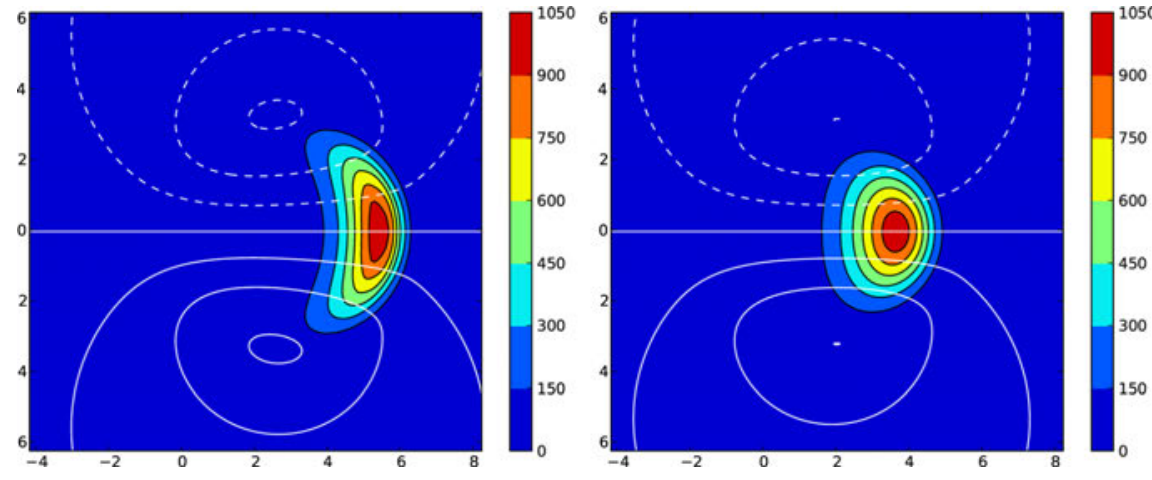

FIG. 4. Particle density contour plots with super imposed electrostatic potential iso lines for $\Delta n / N$ time $t \quad 2$.

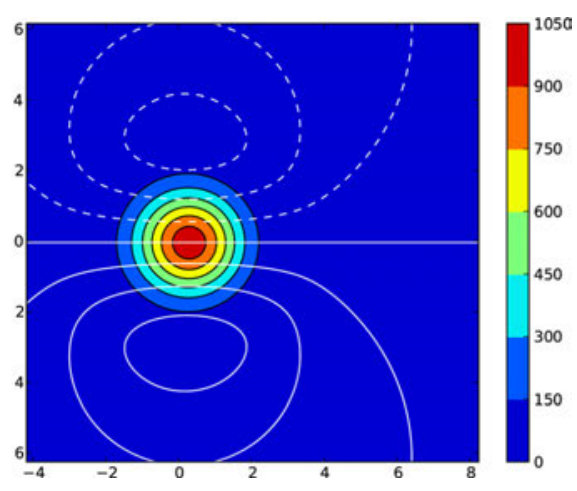

$10^{3}$ and $\nu 10^{1}$ (left), 1 (middle), and 10 (right) at 


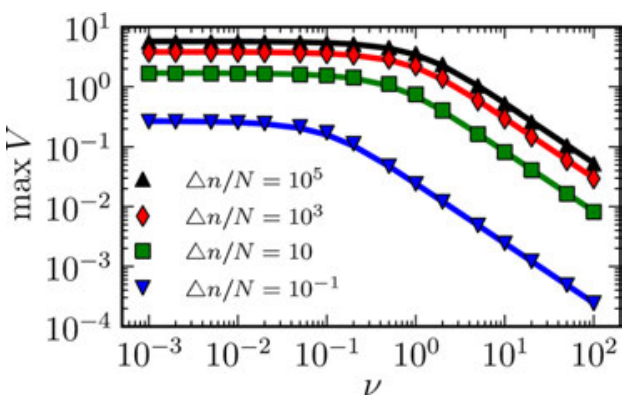

FIG. 5. Maximum radial centre of mass velocity as function of the friction coefficient $\nu$ for various filament amplitudes. The full lines are least square fits of an order of magnitude approximation to the simulation data.

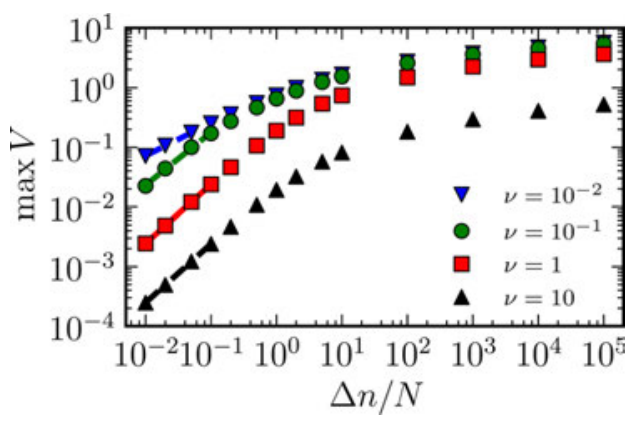

FIG. 6. Maximum radial centre of mass velocity as function of the relative filament amplitude for various friction coefficients. The full lines are least square fits of the simulation data for amplitudes less than unity.

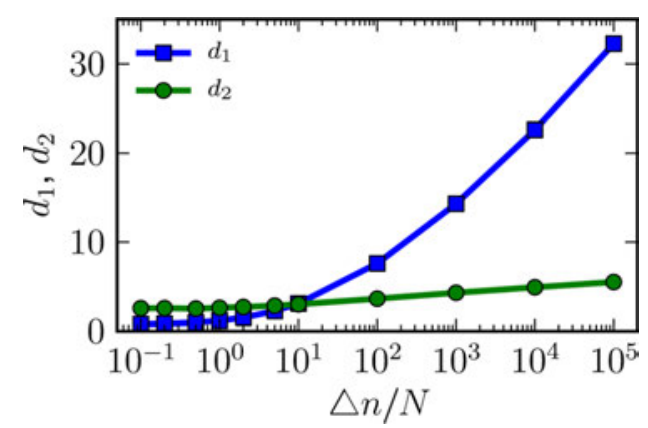

FIG. 7. Fit coefficients $d_{1}$ (square) and $d_{2}$ (circle) for the maximum filament velocity dependence on friction coefficient as function of $\Delta n / N$.

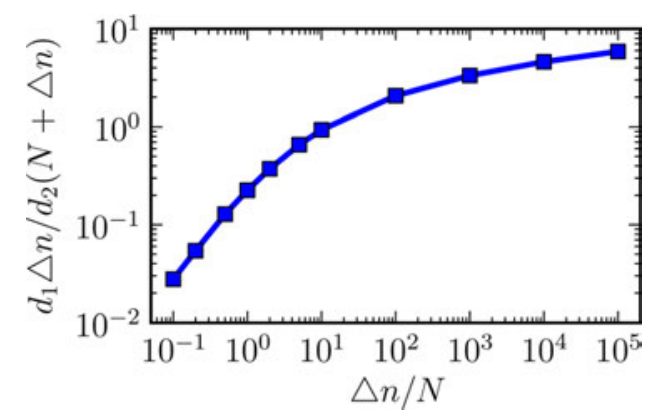

FIG. 8. Amplitude dependence of the maximum radial centre of mass veloc ity $\nu_{\mathrm{i}} V / g$ in the frictional limit.
Note that $d_{1}$ is independent of $\Delta n / N$ for small amplitudes, implying that $V \sim(\Delta n / N)^{1 / 2}$ in this limit. This is consistent with the fit presented in Fig. 6, which gives a scaling exponent of approximately 0.5 for $\nu=10^{2}$ and $\Delta n / N \leq 1$. For large cross-field filament sizes, $\ell \gg g / \nu_{\mathrm{i}}^{2}$, the frictional filament velocity scaling given in Eq. (9) is recovered

$$
V \approx \frac{g}{\nu_{\mathrm{i}}} \frac{d_{1}}{d_{2}} \frac{\Delta n}{N+\Delta n}
$$

For small filament amplitudes this gives a linear dependence on $\Delta n / N$, again consistent with the fitted scaling exponent of approximately unity in Fig. 6 for $\nu \gtrsim 1$ and $\Delta n / N \lesssim 1$.

Except for the numerically determined fit coefficients $d_{1}$ and $d_{2}$, the frictional limit given in Eq. (17) is the same as that found by Ossakow and Chaturvedi for buoyant equatorial irregularities. ${ }^{5}$ Based on an analogy with bubbles in hydrodynamics, Ott arrived at an expression for ESF filament velocities similar to Eq. (15) but without the amplitude dependence. ${ }^{4}$ While the bubble analogy did not reveal the amplitude dependence, it did identify the qualitative size dependence and a transition from the inertial to the frictional velocity scaling regimes. The importance of the amplitude dependence of the maximum centre of mass velocity $\nu_{\mathrm{i}} \mathrm{V} / \mathrm{g}$ in the frictional limit given by Eq. (17) is evident from Fig. 8, where the fit coefficients $d_{1}$ and $d_{2}$ are given in Fig. 7 .

The velocity dependence on filament size given by Eq. (15) is presented in Fig. 9 for a range of amplitudes. It is clearly seen that the radial velocity increases as the square root of the size for small $\ell$ and is independent of size for large $\ell$. The length scale for the transition between these two regimes scales as $g / \nu_{\mathrm{i}}^{2}$ but also depends on the filament amplitude. In order to quantify this amplitude dependence we have calculated the cross-field size which gives the largest curvature for the velocity curves in Fig. 9. In Fig. 10 we present the cross-field size of the transition point between the inertial and frictional velocity scaling regimes. We find that this transition point depends sensitively on the filament amplitude for small amplitudes. For amplitudes $\Delta n / N \gtrsim 10^{2}$ this dependency is weaker and the transition point increases little with increasing filament amplitude. For relative filament amplitudes larger than approximately $10^{3}$ the transition length scale is approximately given by $2 g / \nu_{\mathrm{i}}^{2}$. We conclude that the inertial velocity scaling pertains for larger filament sizes as the amplitude increases.

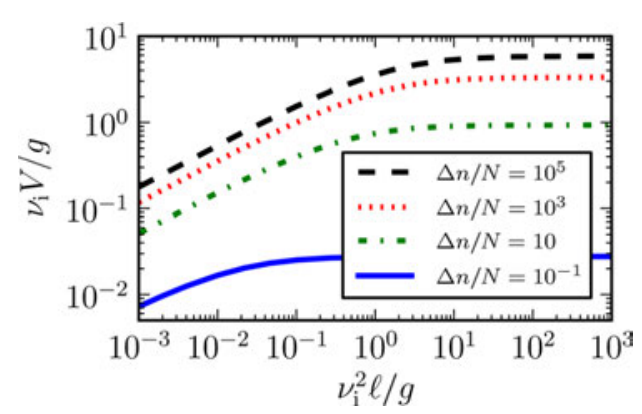

FIG. 9. Dimensional filament velocity as function of the cross field size in the presence of dynamical friction. 


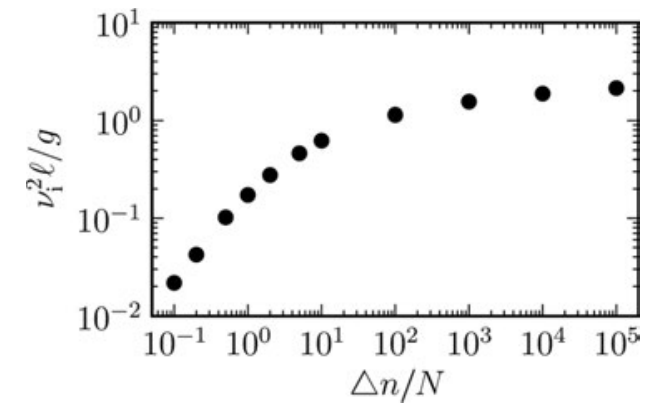

FIG. 10. Cross field filament size that separates the inertial and frictional regimes, given by the point of maximum curvature of $V(\ell)$.

\section{COMPARISON WITH EXPERIMENTS}

The simulation results presented here compare favourably with experimental measurements on the motion of isolated filament structures in the weakly ionized VTF plasmas. ${ }^{8}$ In these experiments, a blob-like filament structure was produced by a pulse of micro waves in a simply magnetized torus configuration. Due to vertical magnetic field gradient and curvature drifts, the toroidally symmetric blob structure becomes polarized and moves radially outwards. Collisions with the neutral Argon gas particles result in loss of momentum. This resembles the dynamics of ESF irregularities and is exactly the situation described by the model and numerical simulations presented here.

In these VTF experiments, it was observed that the bloblike structures in some cases develop a mushroom-like cap shape for the particle density and a dipole electrostatic potential similar to that seen in the numerical simulations. ${ }^{8}$ The radial centre of mass velocity of the filament was calculated from probe measurements and found to be inversely proportional to the neutral gas pressure, with a gas pressure of $10^{4}$ Torr giving a velocity approximately equal to $2 \times 10^{3} \mathrm{~m} / \mathrm{s}$.

Estimates of the ion neutral collision frequency indicate that the VTF blob experiments were in the frictional regime. With the stated electron and ion temperatures of $T_{\mathrm{e}} \approx 2 T_{\mathrm{i}}$ $\approx 2 \mathrm{eV}$, the acoustic speed $C_{\mathrm{s}} \approx 2 \times 10^{3} \mathrm{~m} / \mathrm{s}$. Assuming the neutral gas to be at room temperature and the cross section for ion neutral collisions given by $\sigma \approx 10^{18} \mathrm{~m}^{2}$ (Refs. 34 and 35), the collision frequency $\nu_{\mathrm{i}} \approx n_{\mathrm{g}} \sigma v_{\mathrm{ti}}$ is $9 \times 10^{3} \mathrm{~m} / \mathrm{s}$, where the neutral gas particle density $n_{\mathrm{g}} \approx 3 \times 10^{18} \mathrm{~m}^{3}$ at a pressure of $10^{4}$ Torr. This gives the frictional velocity $g / \nu_{\mathrm{i}} \approx 2 \times 10^{3} \mathrm{~m} / \mathrm{s}$ which is expected to pertain for crossfield filament sizes of the order of $g / \nu_{\mathrm{i}}^{2} \approx 0.2 \mathrm{~m}$ or larger. This is roughly the size of the blob-like structures produced by the solenoid and micro wave pulse in the VTF experiments. We find best agreement between the simulation results and the experimental measurements for a relative filament amplitude of order 10.

For larger neutral gas pressures, the predicted velocity and transition length scale decrease, in accordance with the experimental measurements. For a neutral gas pressure of $5 \times 10^{4}$ Torr the frictional velocity is given by $g / \nu_{\mathrm{i}}$ $\approx 3 \times 10^{2} \mathrm{~m} / \mathrm{s}$, which is just the average filament velocity measured in the experiments. ${ }^{8}$ In this frictional regime, the radial velocity is independent of the cross-field filament size. This was explicitly demonstrated in the VTF experiments where blob structures with different cross-field size were produced by varying the micro wave pulse length. For a given neutral gas pressure, it was found that the radial centre of mass velocity does not change with size. ${ }^{8}$

Despite the overall agreement, it should be noted that there are several uncertainties in the above estimated plasma parameters. First, the low background plasma particle density is not accessible by probe measurements. Thus, the relative filament amplitude, although certainly large, is not exactly known. Second, the ion temperature is not measured in the experiments. Finally, the estimate of the ion-neutral collision frequency is not unambiguous. It should be noted that the transition size $g / \nu_{\mathrm{i}}^{2}$ strongly depends on the friction coefficient. Nevertheless, it is concluded that the prediction of the model and numerical simulations presented here agree well with the VTF experiments.

For blob-like structures in the scrape-off layer of magnetically confined plasmas, we find that ion neutral collisions will strongly influence the filament dynamics provided $\nu \gtrsim 1$, which implies a neutral deuterium particle density of 5 $\times 10^{19} \mathrm{~m}^{3}$ for a filament size $\ell \approx 1 \mathrm{~cm}$, relative amplitude $\Delta n / N \approx 1$, major radius $R \approx 1 \mathrm{~m}$, and equal electron and ion temperatures. Such high neutral particle densities are found in the divertor region and may occur in the scrape-off layer in high recycling or detached plasmas. ${ }^{17-20}$ In the case of ballooned filament structures as discussed in Sec. I and the Appendix, parallel motion with the acoustic or Alfvén speed can lead to effective friction coefficients of the order of 0.1 and 10 , respectively. This indicates that parallel vorticity transport may be important for the filament dynamics. However, numerical simulations of dynamics in all three spatial dimensions are required to self-consistently describe the radial transport associated with the filament motion in this case.

\section{SUMMARY AND CONCLUSIONS}

In summary, we have investigated the radial interchange motion of isolated plasma filaments in the presence of dynamical friction and allowing large relative amplitudes. We have elucidated the filament velocity dependence on crossfield size, amplitude, and plasma parameters in the presence of dynamical friction. Based on an order of magnitude estimate, we have found an analytical expression for this velocity dependence and verified it by numerical simulations.

In the ideal limit there is an inertial velocity scaling, predicting the filament velocity to be proportional to the square root of gravity and the cross-field size. For large friction coefficients, the filament velocity is independent of the cross-field size, proportional to gravity and inversely proportional to the friction coefficient. In this regime, the filament moves a large distance with nearly constant velocity and shape. The crossfield filament size that separates these two regimes is given by the ratio of gravity and the square of the friction coefficient. These results agree with previous theories for buoyancy driven ionospheric irregularities and generalizes them to allow for a large variation of filament amplitudes.

The spatio-temporal filament evolution and predicted velocity scaling are found to compare favourably with measurements of isolated blob-like structures in the VTF device. 
The results presented here agree with (i) the mushroom-like cap shape of the filament structures, (ii) the dipole shaped electrostatic potential, (iii) the inverse scaling of the filament velocity with neutral gas pressure and thereby collision frequency, and (iv) the filament velocity independence on size for strong friction. Moreover, by extending the blob theory and simulations to relative filament amplitudes much larger than unity, we find that the measured blob velocities of the order of the acoustic speed is consistent with theory.

\section{ACKNOWLEDGMENTS}

This work was supported in part by the Norwegian metacentre for computational science (NOTUR) program through grants of computation time. R. Kube was supported with financial subvention from the Research Council of Norway. Discussions with N. Katz, B. LaBombard, B. Lipschultz, B. D. Scott, H. R. Skullerud and J. L. Terry are gratefully appreciated.

\section{APPENDIX: EFFECTIVE FRICTION}

Here we briefly discuss how effective dynamical friction might arise from B-parallel dynamics in the high and low collisionality limits with emphasis on tokamak scrape-off layer plasmas. ${ }^{11,24,25}$ It has been demonstrated in several experiments that the filamentary structures have significant parallel modulation with peak amplitude in the outboard mid-plane region. This so-called ballooning structure leads to flows along the magnetic field that are experimentally measured away from the outboard mid-plane. ${ }^{36-38}$

For simplicity of the argument, let us ignore the crossfield dynamics and focus on the effect of parallel electric currents. The quasi-neutrality condition is then given by

$$
\frac{\rho}{B} \frac{\partial \Omega}{\partial t}=\nabla_{\|} \cdot \mathbf{J}_{\|}
$$

where $\rho$ is the plasma mass density and $\Omega=\nabla_{\perp}^{2} \phi / B$ is the electric drift vorticity. If we average over the parallel length $L$ of the filament structure we obtain

$$
\frac{\partial \nabla_{\perp}^{2} \phi}{\partial t}=\frac{C_{\mathrm{A}}^{2} \mu_{0} J_{\mathrm{w}}}{L}
$$

where $C_{\mathrm{A}}=\left(B^{2} / \mu_{0} \rho\right)^{1 / 2}$ is the Alfvén speed and $J_{\mathrm{w}}$ is the current density at the edge of the filament structure. Since there are no parallel electric fields in the background plasma outside the filament structure, we must have the boundary condition

$$
\nabla_{\|} \phi+\frac{\partial \mathbf{A}_{\|}}{\partial t}=\mathbf{0}
$$

where the electric current perturbations are related to the vector potential by $\mu_{0} J_{\|}=-\nabla_{\perp}^{2} A_{\|}$. For outgoing waves with phase velocity $\omega / k_{\|}=C_{\mathrm{A}}$ it follows that the parallel current is related to the electrostatic potential by $\phi=C_{\mathrm{A}} A_{\|}$. This results in an effective friction term in the field line averaged vorticity equation (A2)

$$
\frac{\partial \nabla_{\perp}^{2} \phi}{\partial t}+\frac{C_{\mathrm{A}}}{L} \nabla_{\perp}^{2} \phi=0
$$

and the effective friction coefficient is evidently given by $\nu_{\mathrm{i}}=C_{\mathrm{A}} / L$. This damping mechanism for interchange motions has previously been used for the description of ablating pellets in tokamak plasmas. ${ }^{39,40}$

Propagation of Alfvén waves will be halted in highly collisional plasmas, such as the scape-off layer in present tokamak experiments with high line-averaged particle density and small plasma currents. Note that if we here include B-parallel motion $\mathbf{V}_{\|} \cdot \nabla$ in the advective derivative in the polarization current given in Eq. (4), we obtain an additional term in the resulting vorticity equation given by $\rho\left(\mathbf{V}_{\|} \cdot \nabla\right) \Omega$. In the collisional limit the parallel advection can be estimated by $C_{\mathrm{s}} / L$, which results in an effective friction term in the vorticity equation with the friction coefficient $\nu_{\mathrm{i}}=C_{\mathrm{s}} / L$. This is the damping term in used in previous twodimensional turbulence simulations of scrape-off layer plasmas. ${ }^{21-23}$ These considerations suggest that the parallel filament dynamics can be parametrized as a simple effective friction in the low and high collisionality limits for strongly ballooned structures. However, three-dimensional numerical simulations are evidently required to reveal the selfconsistent filament dynamics and the transition between these regimes. This is work now in progress.

${ }^{1}$ M. C. Kelley and J. P. McClure, J. Atmos. Terr. Phys. 43, 427 (1981).

${ }^{2}$ S. L. Ossakow, J. Atmos. Terr. Phys. 43, 437 (1981).

${ }^{3}$ D. L. Hysell, J. Atmos. Solar Terr. Phys. 62, 1037 (2000).

${ }^{4}$ E. Ott, J. Geophys. Res. 83, 2066, doi:10.1029/JA083iA05p02066 (1978).

${ }^{5}$ S. L. Ossakow and P. K. Chaturvedi, J. Geophys. Res. 83, 2085, doi:10.1029/JA083iA05p02085 (1978).

${ }^{6}$ F. Øynes, H. L. Pécseli, and K. Rypdal, Phys. Rev. Lett. 75, 81 (1995).

${ }^{7}$ K. Rypdal, O. E. Garcia, and J. V. Paulsen, Phys. Rev. Lett. 79, 1857 (1997).

${ }^{8}$ N. Katz, J. Egedal, W. Fox, A. Le, and M. Porkolab, Phys. Rev. Lett. 101, 015003 (2008).

${ }^{9}$ I. Furno, B. Labit, M. Podestá, A. Fasoli, S. H. Müller, F. M. Poli, P. Ricci, C. Theiler, S. Brunner, A. Diallo, and J. Graves, Phys. Rev. Lett. 100, 055004 (2008).

${ }^{10}$ C. Theiler, I. Furno, P. Ricci, A. Fasoli, B. Labit, S. H. Müller, and G. Plyushchev, Phys. Rev. Lett. 103, 065001 (2009).

${ }^{11}$ S. I. Krasheninnikov, D. A. D'Ippolito, and J. R. Myra, J. Plasma Phys. 74, 679 (2008)

${ }^{12}$ W. Fundamenski, V. Naulin, T. Neukirch, O. E. Garcia, and J. Juul Rasmussen, Plasma Phys. Controlled Fusion 49, R43 (2007).

${ }^{13}$ S. J. Zweben, J. A. Boedo, O. Grulke, C. Hidalgo, B. LaBombard, R. J. Maqueda, P. Scarin, and J. L. Terry, Plasma Phys. Controlled Fusion 49, S1 (2007).

${ }^{14}$ O. E. Garcia, Plasma Fusion Res. 4, 019 (2009).

${ }^{15}$ D. A. D'Ippolito, J. R. Myra, and S. J. Zweben, Phys. Plasmas 18, 060501 (2011).

${ }^{16}$ R. A. Pitts, J. P. Coad, D. P. Coster, G. Federici, W. Fundamenski, J. Horacek, K. Krieger, A. Kukushkin, J. Likonen, G. F. Matthews, M. Rubel, J. D. Strachan, and JET EFDA Contributors, Plasma Phys. Con trolled Fusion 47, B303 (2005).

${ }^{17}$ B. LaBombard, R. L. Boivin, M. Greenwald, J. Hughes, B. Lipschultz, D. Mossessian, C. S. Pitcher, J. L. Terry, S. J. Zweben, and Alcator Group, Phys. Plasmas 8, 2107 (2001).

${ }^{18}$ B. Lipschultz, B. LaBombard, C. S. Pitcher, and R. L. Boivin, Plasma Phys. Controlled Fusion 44, 733 (2002).

${ }^{19}$ B. Lipschultz, D. Whyte, and B. LaBombard, Plasma Phys. Controlled Fusion 47, 1559 (2005).

${ }^{20}$ B. LaBombard, J. L. Terry, J. W. Hughes, D. Brunner, J. Payne, M. L. Reinke, I. Cziegler, R. Granetz, M. Greenwald, I. H. Hutchinson, J. Irby, Y. Lin, B. Lipschultz, Y. Ma, E. S. Marmar, W. L. Rowan, N. Tsujii, 
G. Wallace, D. G. Whyte, S. Wolfe, S. Wukitch, G. Wurden, and Alcator C Mod Team, Phys. Plasmas 18, 056104 (2011).

${ }^{21}$ O. E. Garcia, J. Horacek, R. A. Pitts, A. H. Nielsen, W. Fundamenski, J. P. Graves, V. Naulin, and J. J. Rasmussen, Plasma Phys. Controlled Fusion 48, L1 (2006); Nucl. Fusion 47, 667 (2007); J. Nucl. Mater. 363-365, 575 (2007).

${ }^{22}$ W. Fundamenski, O. E. Garcia, V. Naulin, R. A. Pitts, A. H. Nielsen, J. J. Rasmussen, J. Horacek, J. P. Graves, and JET EFDA Contributors, Nucl. Fusion 47, 417 (2007).

${ }^{23}$ O. E. Garcia, V. Naulin, A. H. Nielsen, and J. J. Rasmussen, Phys. Rev. Lett. 92, 165003 (2004); Phys. Plasmas 12, 062309 (2005); Phys. Scr. T122, 89 (2006).

${ }^{24}$ S. I. Krasheninnikov, A. I. Smolyakov, G. Yu, and T. K. Soboleva, Phys. Scr. T124, 13 (2006)

${ }^{25}$ S. I. Krasheninnikov and A. I. Smolyakov, Phys. Plasmas 15, 055909 (2008).

${ }^{26}$ O. E. Garcia, N. H. Bian, V. Naulin, A. H. Nielsen, and J. Juul Rasmussen, Phys. Scr. T122, 104 (2006).

${ }^{27}$ M. N. Rosenbluth and C. L. Longmire, Ann. Phys. 1, 120 (1957).

${ }^{28}$ O. E. Garcia, J. Plasma Phys. 65, 81 (1999); Eur. J. Phys. 24, 331 (2003).

${ }^{29}$ S. I. Krasheninnikov, Phys. Lett. A 283, 368 (2001).

${ }^{30}$ D. A. D’Ippolito, J. R. Myra, and S. I. Krasheninnikov, Phys. Plasmas 9, 222 (2002).

${ }^{31}$ O. E. Garcia, N. H. Bian, V. Naulin, A. H. Nielsen, and J. J. Rasmussen, Phys. Plasmas 12, 090701 (2005).
${ }^{32}$ O. E. Garcia, N. H. Bian, and W. Fundamenski, Phys. Plasmas 13, 082309 (2006).

${ }^{33}$ R. Kube and O. E. Garcia, Phys. Plasmas 18, 102314 (2011).

${ }^{34}$ R. Hegerberg, M. T. Elford, and H. R. Skullerud, J. Phys. B: At. Mol. Opt. Phys. 15, 797 (1982).

${ }^{35}$ A. V. Phelps, J. Phys. Chem. Ref. Data 20, 557 (1991); J. Appl. Phys. 76, 747 (1994).

${ }^{36}$ B. LaBombard, J. E. Rice, A. E. Hubbard, J. W. Hughes, M. Greenwald, J. Irby, Y. Lin, B. Lipschultz, E. S. Marmar, C. S. Pitchera, N. Smick, S. M Wolfe, S. J. Wukitch, and the Alcator Group, Nucl. Fusion 44, 1047 (2004).

${ }^{37}$ R. A. Pitts, J. Horacek, W. Fundamenski, O. E. Garcia, A. H. Nielsen, M. Wischmeier, V. Naulin, and J. J. Rasmussen, J. Nucl. Mater. 363-365, 505 (2007).

${ }^{38}$ J. P. Gunn, C. Boucher, M. Dionne, I. Duran, V. Fuchs, T. Loarer, I. Nano bashvili, R. Pánek, J. Y. Pascal, F. Saint Laurent, J. Stöckel, T. Van Rom puy, R. Zagórski, J. Adámek, J. Bucalossi, R. Dejarnac, P. Devynck, P. Hertout, M. Hron, G. Lebrun, P. Moreau, F. Rimini, A. Sarkissian, and G. Van Oost, J. Nucl. Mater. 363-365, 484 (2007).

${ }^{39}$ P. B. Parks, W. D. Sessions, and L. R. Baylor, Phys. Plasmas 7, 1968 (2000).

${ }^{40}$ V. Rozhansky, I. Senichenkov, I. Veselova, and R. Schneider, Plasma Phys. Controlled Fusion 46, 575 (2004). 